\title{
LERAY'S SELF-SIMILAR SOLUTIONS TO THE NAVIER-STOKES EQUATIONS WITH PROFILES IN MARCINKIEWICZ AND MORREY SPACES
}

\author{
CRISTI GUEVARA AND NGUYEN CONG PHUC*
}

\begin{abstract}
We rule out the existence of Leray's backward self-similar solutions to the Navier-Stokes equations with profiles in $L^{12 / 5}\left(\mathbb{R}^{3}\right)$ or in the Marcinkiewicz space $L^{q, \infty}\left(\mathbb{R}^{3}\right)$ for $q \in(12 / 5,6)$. This follows from a more general result formulated in terms of Morrey spaces and the first order Riesz's potential.
\end{abstract}

\section{INTRODUCTION}

The motion of an incompressible fluid in three spatial dimensions, $\mathbb{R}^{3}$, with viscosity $\nu>0$ and zero external force is described by the NavierStokes equations

$$
\left\{\begin{array}{c}
u_{t}-\nu \Delta u+u \cdot \nabla u+\nabla p=0 \\
\operatorname{div} u=0
\end{array}\right.
$$

with an initial condition $u(x, 0)=u_{0}(x)$. Here the unknown velocity $u=$ $u(x, t)=\left(u_{1}(x, t), u_{2}(x, t), u_{3}(x, t)\right) \in \mathbb{R}^{3}$ and the unknown pressure $p=$ $p(x, t) \in \mathbb{R}$ are defined for each position $x \in \mathbb{R}^{3}$ and time $t \geq 0$.

Since Leray's work 10] in 1934, there has been a long-standing question whether solutions to (1.1) develop a singularity in finite time, or whether (1.1) admits a time-global smooth solution for any given smooth and compactly supported initial datum $u_{0}$. To look for a singular solution, Leray [10] suggested to consider the backward self-similar solutions of (1.1), i.e., those of the form

$$
u(x, t)=\lambda(t) U(\lambda(t) x), \quad p(x, t)=\lambda^{2}(t) P(\lambda(t) x),
$$

where

$$
\lambda(t)=\frac{1}{\sqrt{2 a(T-t)}}, \quad a>0, \quad T>0,
$$

and $U=\left(U_{1}, U_{2}, U_{3}\right)$ and $P$ are defined in the whole $\mathbb{R}^{3}$. Note then that $u$ is defined in $\mathbb{R}^{3} \times(-\infty, T)$ and if the profile $U$ is not identically zero then $u$ given by (1.2) develops a singularity at time $t=T$. Here certain natural energy norms of $u$ should be required to be finite. For otherwise the profile $U=\nabla \Phi$ and $P=-\frac{1}{2}|U|^{2}-a y \cdot U$, for any non-zero harmonic function $\Phi$, would immediately yield a non-trivial self-similar solution.

${ }^{*}$ Supported in part by the Simons Foundation, Award Number: 426071. 
By direct calculations, one finds that $(u, p)$ of the form (1.2) is a solution of (1.1) if and only if $(U, P)$ solves the following nonlinear time-independent system in $\mathbb{R}^{3}$ :

$$
\left\{\begin{array}{c}
-\nu \Delta U+a U+a(y \cdot \nabla) U+(U \cdot \nabla) U+\nabla P=0 \\
\operatorname{div} U=0
\end{array}\right.
$$

Leray's question was open until 1996 when Nečas, Růžička, and Šverák 12 showed that there does not exist a non-trivial solution of the form (1.2) with finite kinetic energy and satisfies the natural global energy inequality

$$
\int_{\mathbb{R}^{3}} \frac{1}{2}|u(x, t)|^{2} d x+\int_{t_{1}}^{t} \int_{\mathbb{R}^{3}} \nu|\nabla u(x, t)|^{2} d x d t \leq \int_{\mathbb{R}^{3}} \frac{1}{2}\left|u\left(x, t_{1}\right)\right|^{2} d x
$$

for all $t \in\left(t_{1}, t_{2}\right)$. More generally, they proved that if $U \in W_{\text {loc }}^{1,2}\left(\mathbb{R}^{3}\right) \cap L^{3}\left(\mathbb{R}^{3}\right)$ is a weak solution of (1.3) then $U \equiv 0$. Note that the $L^{3}$ global integrability condition of $U$ holds if the corresponding self-similar solution $u$ satisfies the global energy estimates (1.4).

On the other hand, [12] left open the question of existence of self-similar singularities which satisfy only the local energy inequality

$$
\underset{t_{3}<t<T}{\operatorname{ess} \sup _{B_{r}\left(x_{0}\right)}} \frac{1}{2}|u(x, t)|^{2} d x+\int_{t_{3}}^{T} \int_{B_{r}\left(x_{0}\right)} \nu|\nabla u(x, t)|^{2} d x d t<+\infty,
$$

for some ball $B_{r}\left(x_{0}\right)$ and some $t_{3}<T$. This question was later answered by Tsai in [18, where he showed that backward self-similar solutions to (1.1) satisfying (1.5) must also be zero:

Theorem 1.1 (Tsai [18]). Suppose $u$ is a weak solution of (1.1) satisfying the finite local energy condition (1.5) for some ball $B_{r}\left(x_{0}\right)$ and some $t_{3}<T$. If $u$ is of the form (1.2), then $u \equiv 0$.

In the same paper Tsai also extended the result of [12] to a super critical range of the integrability condition on the profile $U$ :

Theorem 1.2 (Tsai [18]). If a weak solution $U \in W_{\text {loc }}^{1,2}\left(\mathbb{R}^{3}\right)$ of (1.3) belongs $L^{q}\left(\mathbb{R}^{3}\right)$ for some $q \in(3, \infty]$ then it must be zero provided $q \neq \infty$ and constant provided $q=\infty$.

The proofs of Theorems 1.1 and 1.2 use the important fact that the scalar function

$$
\Pi(y)=\frac{1}{2}|U(y)|^{2}+P(y)+a y \cdot U(y)
$$

satisfies the maximum principle. This idea had been employed earlier in the work of Nečas, Rǔžička, and Šverák [12] to treat the critical case $q=3$ mentioned above. See also the earlier work [3, 4, 19] in the context of stationary Navier-Stokes equations in higher dimensions.

We mention that the proof of Theorem 1.1 in [18 also makes use of a celebrated $\epsilon$-regularity criterion due to Caffarelli, Kohn, and Nirenberg [2] to show that if $(u, p)$ of the form (1.2) is a suitable weak solution of (1.1) in $B_{1}(0) \times(T-1, T)$ then $U(y)=O\left(|y|^{-1}\right)$ as $y \rightarrow \infty$ (see [18, Corollary 4.3]). 
On the other hand, one important step in Tsai's proof of Theorem 1.2 is to show that if $U$ is a weak solution of (1.3) and $U \in L^{q}\left(\mathbb{R}^{3}\right)$ for some $3<q<\infty$ then $U=o(|y|)$ as $y \rightarrow \infty$ (see [18, Lemma 3.3]). He also remarked that his approach to this pointwise asymptotic estimate fails at the end-point case $q=3$ and suggested that the sub-critical case $q<3$ would require a different idea (see [18, Remark 3.2]).

A main goal of this paper is to improve the result of 12 by allowing the profile $U$ to be in spaces strictly larger than $L^{3}\left(\mathbb{R}^{3}\right)$. Furthermore, by that way we also extend the result of Theorem 1.2 to the sub-critical range $q \in\left[\frac{12}{5}, 3\right)$. Indeed, we prove

Theorem 1.3. Let $U \in W_{\text {loc }}^{1,2}\left(\mathbb{R}^{3}\right)$ be a weak solution of (1.3). If $U \in$ $L^{q, \infty}\left(\mathbb{R}^{3}\right)$ for some $q \in\left(\frac{12}{5}, 6\right)$ or if $U \in L^{\frac{12}{5}}\left(\mathbb{R}^{3}\right)$ then it must be identically zero.

In the above theorem, the space $L^{q, \infty}\left(\mathbb{R}^{3}\right)$ is a the Marcinkiewicz space (or weak $L^{q}$ space) defined as the set of measurable functions $g$ in $\mathbb{R}^{3}$ such that the quasinorm

$$
\|g\|_{L^{q, \infty}\left(\mathbb{R}^{3}\right)}:=\sup _{\alpha>0} \alpha\left|\left\{x \in \mathbb{R}^{3}:|g(x)|>\alpha\right\}\right|^{\frac{1}{q}}<+\infty .
$$

It is well-known that $L^{q}\left(\mathbb{R}^{3}\right) \subset L^{q, \infty}\left(\mathbb{R}^{3}\right)$, or more generally $L^{q, s}\left(\mathbb{R}^{3}\right) \subset$ $L^{q, \infty}\left(\mathbb{R}^{3}\right)$ for any $s>0$, where $L^{q, s}\left(\mathbb{R}^{3}\right), q>0, s \in(0, \infty)$, is the Lorentz space with quasinorm

$$
\|g\|_{L^{q, s}\left(\mathbb{R}^{3}\right)}:=\left(q \int_{0}^{\infty} \alpha^{s}\left|\left\{x \in \mathbb{R}^{3}:|g(x)|>\alpha\right\}\right|^{\frac{s}{q}} \frac{d \alpha}{\alpha}\right)^{\frac{1}{s}} .
$$

Note that $L^{q}\left(\mathbb{R}^{3}\right)=L^{q, q}\left(\mathbb{R}^{3}\right)$ and $L^{q, s_{1}}\left(\mathbb{R}^{3}\right) \subset L^{q, s_{2}}\left(\mathbb{R}^{3}\right)$ for $0<s_{1} \leq s_{2}$.

Recently in Phuc [14], it is shown that locally finite energy solutions to the Navier-Stokes equation (1.1) belonging to $L_{t}^{\infty}\left(L_{x}^{3, s}\right)$ are regular provided $s \neq \infty$. That result strengthens the above mentioned result of $[12$ as it rules out the existence of self-similar solutions of the form (1.2) with profiles $U \in L^{3, s}\left(\mathbb{R}^{3}\right)$ provided $s \neq \infty$. Thus, in the case $q=3$ Theorem 1.3 provides the answer to the end-point case $s=\infty$. Note on the other hand that it is still unknown whether $L_{t}^{\infty}\left(L_{x}^{3, \infty}\right)$ solutions to the Navier-Stokes equations are regular.

In fact, we shall prove a more general result than Theorem 1.3 which allows the profile $U$ to have a very modest decay at infinity. To describe it, recall that the Riesz potential $\mathbf{I}_{\alpha}, \alpha \in(0,3)$, on $\mathbb{R}^{3}$ is defined by

$$
\mathbf{I}_{\alpha} f(x)=c(\alpha) \int_{\mathbb{R}^{3}} \frac{f(y)}{|x-y|^{3-\alpha}} d y, \quad x \in \mathbb{R}^{3},
$$

for $f \in L_{\text {loc }}^{1}\left(\mathbb{R}^{3}\right)$ such that $\int_{|x| \geq 1}|x|^{\alpha-3}|| f(x) \mid d x<+\infty$. Here the normalizing constant

$$
c(\alpha)=\frac{\Gamma\left(\frac{3}{2}-\frac{\alpha}{2}\right)}{\pi^{3 / 2} 2^{\alpha} \Gamma(\alpha / 2)} .
$$


Additionally, we define the Morrey space $\mathcal{M}^{p, \gamma}\left(\mathbb{R}^{3}\right), p \geq 1,0<\gamma \leq 3$, to be the set of all functions $f \in L_{\text {loc }}^{p}\left(\mathbb{R}^{3}\right)$ such that

$$
\int_{B_{r}(x)}|f(y)|^{p} d y \leq C r^{3-\gamma},
$$

for all $x \in \mathbb{R}^{3}$ and $r>0$ with a constant $C$ independent of $x$ and $r$. The norm $\|f\|_{\mathcal{M}^{p, \gamma}\left(\mathbb{R}^{3}\right)}$ is given by

$$
\|f\|_{\mathcal{M}^{p, \gamma\left(\mathbb{R}^{3}\right)}}:=\sup _{x \in \mathbb{R}^{3}, r>0} r^{\frac{\gamma-3}{p}}\|f\|_{L^{p}\left(B_{r}(x)\right)} .
$$

Obviously, when $\gamma=3$ we have $\mathcal{M}^{p, \gamma}\left(\mathbb{R}^{3}\right)=L^{p}\left(\mathbb{R}^{3}\right)$. The interest of using such a notation for Morrey spaces is to emphasize that the second index $\gamma$ acts like the dimension in the Sobolev type embedding theorem. Indeed, it is now well-known from the work of D. R. Adams [1] that $\mathbf{I}_{\alpha}$ continuously maps $\mathcal{M}^{p, \gamma}\left(\mathbb{R}^{3}\right)$ into $\mathcal{M}^{\frac{\gamma p}{\gamma-\alpha p}, \gamma}\left(\mathbb{R}^{3}\right)$ provided $1<p<\gamma / \alpha$. Thus when $\gamma=3$ the classical Sobolev embedding theorem is recovered.

We are now ready to state the next result of the paper:

Theorem 1.4. Let $U \in W_{\mathrm{loc}}^{1,2}\left(\mathbb{R}^{3}\right)$ be a weak solution of (1.3). If for some $\gamma \in(0,3]$ it holds that

$$
\int_{B_{r}} \mathbf{I}_{1}\left(\chi_{B_{r}}|U|^{2}\right)^{2} d x \leq C r^{3-\gamma} \quad \forall \text { balls } B_{r} \subset \mathbb{R}^{3},
$$

where $\chi_{B_{r}}$ is the characteristic function of $B_{r}$, then $U \equiv 0$. In particular, if $\mathbf{I}_{1}\left(|U|^{2}\right) \in \mathcal{M}^{2, \gamma}\left(\mathbb{R}^{3}\right)$ for some $\gamma \in(0,3]$ then $U$ must be identically zero.

We now show that Theorem 1.4 actually implies Theorem 1.3. Indeed, if $U \in L^{12 / 5}\left(\mathbb{R}^{3}\right)$ then the Sobolev embedding theorem implies that $\mathbf{I}_{1}\left(|U|^{2}\right) \in$ $L^{2}\left(\mathbb{R}^{3}\right)=\mathcal{M}^{2,3}\left(\mathbb{R}^{3}\right)$. Thus by Theorem 1.4 , if $U$ is also a weak solution of (1.3) then $U \equiv 0$. Similarly, if $U \in L^{q, \infty}\left(\mathbb{R}^{3}\right)$ with $q \in\left(\frac{12}{5}, 6\right)$ then $\mathbf{I}_{1}\left(|U|^{2}\right) \in L^{\frac{3 q}{6-q}, \infty}\left(\mathbb{R}^{3}\right)$. Since $q>12 / 5$ it follows that $\frac{3 q}{6-q}>2$ and thus by Hölder's inequality we have

$$
\int_{B_{r}(x)} \mathbf{I}_{1}\left(|U|^{2}\right)^{2} d y \leq C\left\|\mathbf{I}_{1}\left(|U|^{2}\right)\right\|_{L^{3 q /(6-q), \infty}\left(\mathbb{R}^{3}\right)}^{2} r^{3-\frac{2(6-q)}{q}} .
$$

This yields $\mathbf{I}_{1}\left(|U|^{2}\right) \in \mathcal{M}^{2, \gamma}\left(\mathbb{R}^{3}\right)$ with $\gamma=\frac{2(6-q)}{q} \in(0,3)$, and thus Theorem 1.3 is a consequence of Theorem 1.4.

On the other hand, using Adams Embedding Theorem [1] we have another corollary of Theorem 1.4.

Corollary 1.5. If $U \in W_{\mathrm{loc}}^{1,2}\left(\mathbb{R}^{3}\right)$ is a weak solution of (1.3) such that if $U \in \mathcal{M}^{\frac{4 \gamma}{2+\gamma}, \gamma}\left(\mathbb{R}^{3}\right)$ for some $\gamma \in(2,3]$ (or equivalently if $U \in \mathcal{M}^{p, \frac{2 p}{4-p}}\left(\mathbb{R}^{3}\right.$ ) for some $p \in(2,12 / 5])$, then $U \equiv 0$.

The proof of Theorem 1.4 will be given in Section 4. Surprisingly, it is based on an application of Theorem 1.1 above. For that a pressure profile 
$P$ is built from $U$ so that the norm of $P$ in a Sobolev space of negative order (localized in each ball) is well controlled. Here one has to treat $P$ as a signed distribution in $\mathbb{R}^{3}$ as no control of $|P|$ is available. This suggests a natural way to control the nonlinear and the pressure terms in the energy equality, and a sort of bootstrapping argument based on the energy equality eventually completes the proof.

\section{Preliminaries}

Throughout the paper we denote by $B_{r}(x)$ the open ball centered at $x \in \mathbb{R}^{3}$ with radius $r>0$, i.e.,

$$
B_{r}(x)=\left\{y \in \mathbb{R}^{3}:|x-y|<r\right\} .
$$

We write $\partial_{j} u_{i}=\frac{\partial u_{i}}{\partial x_{j}}$ and use the letters $C$ or $c$ to denote generic constants that could be different from line to line.

For each bounded open set $O \subset \mathbb{R}^{3}$, we denote by $L^{-1,2}(O)$ the dual of the Sobolev space $W_{0}^{1,2}(O)$. The latter is defined as the completion of $C_{0}^{\infty}(O)$ (the space of smooth functions with compact support in $O$ ) under the Dirichlet integral

$$
\|\varphi\|_{W_{0}^{1,2}(O)}=\left(\int_{O}|\nabla \varphi|^{2} d x\right)^{\frac{1}{2}} .
$$

We shall use the following well-known representation of a function $\varphi \in$ $C_{0}^{\infty}\left(\mathbb{R}^{3}\right)$

$$
\varphi(x)=\frac{1}{\left|S^{2}\right|} \int_{\mathbb{R}^{3}} \frac{(x-y) \cdot \nabla \varphi(y)}{|x-y|^{3}} d y,
$$

where $\left|S^{2}\right|$ is the area of the unit sphere (see, e.g., [15, p. 125]). Identity (2.1) can be used to show that

$$
\|f\|_{L^{-1,2}(O)} \leq C\left\|\mathbf{I}_{1}\left(\chi_{O}|f|\right)\right\|_{L^{2}(O)}
$$

for any $f \in L_{\text {loc }}^{1}\left(\mathbb{R}^{3}\right)$ and any bounded open set $O \subset \mathbb{R}^{3}$. Indeed, for any $\varphi \in C_{0}^{\infty}(O)$ by (2.1) it holds that

$$
\begin{aligned}
\left|\int_{O} \varphi(x) f(x) d x\right| & \leq C \int_{O}\left[\int_{O} \frac{|\nabla \varphi(y)|}{|x-y|^{2}} d y\right]|f(x)| d x \\
& =C \int_{O}|\nabla \varphi(y)|\left[\int_{O} \frac{|f(x)| d x}{|x-y|^{2}}\right] d y \\
& \leq C\|\nabla \varphi\|_{L^{2}(O)}\left\|\mathbf{I}_{1}\left(\chi_{O}|f|\right)\right\|_{\left.L^{2}(O)\right)}
\end{aligned}
$$

as desired.

The following lemma will be needed later. Its proof is based on a simple iteration and can be found in [7, Lemma 6.1]. 
Lemma 2.1. Let $I(s)$ be a bounded nonnegative function in the interval $\left[R_{1}, R_{2}\right]$. Assume that for every $s, \rho \in\left[R_{1}, R_{2}\right]$ and $s<\rho$ we have

$$
I(s) \leq\left[A(\rho-s)^{-\alpha}+B(\rho-s)^{-\beta}+C\right]+\theta I(\rho)
$$

with $A, B, C \geq 0, \alpha>\beta>0$ and $\theta \in[0,1)$. Then it holds that

$$
I\left(R_{1}\right) \leq c(\alpha, \theta)\left[A\left(R_{2}-R_{1}\right)^{-\alpha}+B\left(R_{2}-R_{1}\right)^{-\beta}+C\right] .
$$

We now make precise the definition of a weak solution to the system (1.3).

Definition 2.2. A divergence-free vector field $U=\left(U_{1}, U_{2}, U_{3}\right)$ is called a weak solution of (1.3) if $U \in W_{\text {loc }}^{1,2}\left(\mathbb{R}^{3}\right)$ and if for all divergence-free vector field $\phi=\left(\phi_{1}, \phi_{2}, \phi_{3}\right) \in C_{0}^{\infty}\left(\mathbb{R}^{3}\right)$ one has

$$
\int_{\mathbb{R}^{3}}(\nu \nabla U \cdot \nabla \phi+[a U+a(y \cdot \nabla) U+(U \cdot \nabla) U] \cdot \phi) d y=0 .
$$

\section{THE PRESSURE FORMULATION}

It is known that every weak solution $U$ of (1.3) is smooth (see [5, 6, 9, 17]). Note that Definition 2.2 does not include a pressure $P$. However, taking the divergence of (1.3) we formally obtain the pressure equation 1

$$
-\Delta P=\partial_{i} \partial_{j}\left(U_{i} U_{j}\right) \text {. }
$$

The main goal of this section is to recover a $P$ (with a useful control) from a weak solution $U$ of (1.3) for which (1.6) holds. As $P$ is generally a signed distribution, an estimate of the form (1.6) should not hold if $|U|^{2}$ is replaced by $|P|$. On the other hand, we observe that $(1.6)$ is equivalent to the condition

$$
\left\||U|^{2}\right\|_{L^{-1.2\left(B_{r}\right)}}^{2} \leq C r^{3-\gamma} \quad \forall \text { balls } B_{r} \subset \mathbb{R}^{3},
$$

for some $\gamma \in(0,3]$. Thus it is natural to expect that the pressure $P$ should also satisfy a similar condition in which $|U|^{2}$ is replaced by $P$.

To construct such a $P$ we start with the following lemma.

Lemma 3.1. Let $U=\left(U_{1}, U_{2}, U_{3}\right)$ satisfy (1.6) for some $\gamma \in(0,3]$. For each $i, j \in\{1,2,3\}$ there exists a vector field $G_{i j} \in \mathcal{M}^{2, \gamma}\left(\mathbb{R}^{3}\right)$ such that

$$
-\operatorname{div} G_{i j}=U_{i} U_{j}
$$

in the sense of distributions, i.e.,

$$
\int_{\mathbb{R}^{3}} G_{i j} \cdot \nabla \varphi d x=\int_{\mathbb{R}^{3}} U_{i} U_{j} \varphi d x
$$

for all $\varphi \in C_{0}^{\infty}\left(\mathbb{R}^{3}\right)$.

\footnotetext{
${ }^{1}$ Here and in what follows we use the usual convention and sum over the repeated indices.
} 
Proof. By hypothesis, we see that for every $i, j \in\{1,2,3\}$,

$$
\int_{B_{r}} \mathbf{I}_{1}\left(\chi_{B_{r}}\left|U_{i} U_{j}\right|\right)^{2} d x \leq C r^{3-\gamma}, \quad \forall B_{r} \subset \mathbb{R}^{3} .
$$

Let $\left\{\Psi_{N}(x)\right\}$ be a sequence of smooth functions in $\mathbb{R}^{3}$ such that $0 \leq \Psi_{N} \leq$ $1, \Psi_{N}(x)=1$ for $|x| \leq N / 2, \Psi_{N}(x)=0$ for $|x| \geq N$, and $\left|\nabla \Psi_{N}(x)\right| \leq c / N$. Thus we also have

$$
\int_{B_{r}} \mathbf{I}_{1}\left(\chi_{B_{r}} \Psi_{N}\left|U_{i} U_{j}\right|\right)^{2} d x \leq C r^{3-\gamma}, \quad \forall B_{r} \subset \mathbb{R}^{3},
$$

which by (2.2) yields

$$
\left\|\Psi_{N} U_{i} U_{j}\right\|_{L^{-1,2}\left(B_{r}\right)} \leq C r^{\frac{3-\gamma}{2}}, \quad \forall B_{r} \subset \mathbb{R}^{3},
$$

where $C$ is independent of $N$.

For each $N \geq 1$, we claim that there exists a vector field $G_{i j}^{N} \in \mathcal{M}^{2, \gamma}\left(\mathbb{R}^{3}\right)$ such that

$$
-\operatorname{div} G_{i j}^{N}=\Psi_{N} U_{i} U_{j}
$$

and

$$
G_{i j}^{N} \rightarrow G_{i j} \text { strongly in } \mathcal{M}^{2, \gamma}\left(\mathbb{R}^{3}\right)
$$

as $N \rightarrow+\infty$ for some vector field $G_{i j}$. In particular, $\lim _{N \rightarrow \infty}\left\langle G_{i j}^{N}\right.$, $\left.\phi\right\rangle=$ $\left\langle G_{i j}, \phi\right\rangle$ for every vector field $\phi \in C_{0}^{\infty}\left(\mathbb{R}^{3}\right)$. In view of (3.4), this gives (3.2) as as desired.

Thus it is left to show (3.4) and (3.5). To that end, we define

$$
G_{i j}^{N}(x)=\frac{1}{\left|S^{2}\right|} \int_{\mathbb{R}^{3}} \frac{y-x}{|y-x|^{3}} \Psi_{N}(y) U_{i}(y) U_{j}(y) d y .
$$

Note that $G_{i, j}^{N}=\nabla(-\Delta)^{-1}\left(\Psi_{N} U_{i} U_{j}\right)$ in the sense of distributions, i.e.,

$$
\left\langle G_{i j}^{N}, \phi\right\rangle:=\left\langle\Psi_{N} U_{i} U_{j},-\operatorname{div} \mathbf{I}_{2}(\phi)\right\rangle=\left\langle U_{i} U_{j},-\Psi_{N} \operatorname{div} \mathbf{I}_{2}(\phi)\right\rangle
$$

for $\phi=\left(\phi_{1}, \phi_{2}, \phi_{3}\right) \in C_{0}^{\infty}\left(\mathbb{R}^{3}\right)$. Then obviously, (3.4) holds in the sense of distributions.

On the other hand, for every $k \in\{1,2,3\}, N, M \geq 1$, and every scalar function $\varphi \in C_{0}^{\infty}\left(B_{r}\right)$ we have

$$
\left\langle\left(\Psi_{N}-\Psi_{M}\right) U_{i} U_{j}, \partial_{k} \mathbf{I}_{2}(\varphi)\right\rangle=\sum_{\nu=0}^{\infty}\left\langle\left(\Psi_{N}-\Psi_{M}\right) U_{i} U_{j}, \eta_{\nu} \partial_{k} \mathbf{I}_{2}(\varphi)\right\rangle,
$$

where $\left\{\eta_{\nu}\right\}_{\nu=0}^{\infty}$ is a smooth partition of unity associated to the ball $B_{r}$. That is, $\eta_{0} \in C_{0}^{\infty}\left(B_{2 r}\right), \eta_{\nu} \in C_{0}^{\infty}\left(B_{2^{\nu+1} r} \backslash B_{2^{\nu-1} r}\right), \nu=1,2,3, \ldots$, such that

$$
0 \leq \eta_{\nu} \leq 1, \quad\left|\nabla \eta_{\nu}\right| \leq c\left(2^{j} r\right)^{-1}, \quad \nu=0,1,2, \ldots,
$$

and

$$
\sum_{\nu=0}^{\infty} \eta_{\nu}(x)=1, \quad x \in \mathbb{R}^{3}
$$


Note that the sum in (3.6) has only a finite number of non-zero terms. Moreover, by the property of $\left\{\Psi_{N}\right\}$ we have

$$
\left\langle\left(\Psi_{N}-\Psi_{M}\right) U_{i} U_{j}, \partial_{k} \mathbf{I}_{2}(\varphi)\right\rangle=\sum_{\nu=N_{0}}^{\infty}\left\langle\left(\Psi_{N}-\Psi_{M}\right) U_{i} U_{j}, \eta_{\nu} \partial_{k} \mathbf{I}_{2}(\varphi)\right\rangle,
$$

where $N_{0} \rightarrow+\infty$ as $M, N \rightarrow+\infty$. Using this and (3.3), we have

$$
\begin{aligned}
& \left|\left\langle\left(\Psi_{N}-\Psi_{M}\right) U_{i} U_{j}, \partial_{k} \mathbf{I}_{2}(\varphi)\right\rangle\right| \\
& \leq c \sum_{\nu=N_{0}}^{\infty}\left(2^{\nu} r\right)^{\frac{3-\gamma}{2}}\left\|\nabla\left[\eta_{\nu} \partial_{k} \mathbf{I}_{2}(\varphi)\right]\right\|_{L^{2}\left(B_{2^{\nu+1}}\right)} \\
& \leq c \sum_{\nu=N_{0}}^{\infty}\left(2^{\nu} r\right)^{\frac{3-\gamma}{2}} 2^{-\nu \frac{3}{2}}\|\varphi\|_{L^{2}\left(B_{r}\right)} \\
& \leq c r^{\frac{3-\gamma}{2}}\|\varphi\|_{L^{2}\left(B_{r}\right)} \sum_{\nu=N_{0}}^{\infty} 2^{-\frac{\nu \gamma}{2}} .
\end{aligned}
$$

Here in (3.7) we used the bound

$$
\left\|\nabla\left[\eta_{\nu} \partial_{k} \mathbf{I}_{2}(\varphi)\right]\right\|_{L^{2}\left(B_{2^{\nu+1} 1_{r}}\right)} \leq c 2^{-\nu \frac{3}{2}}\|\varphi\|_{L^{2}\left(B_{r}\right)},
$$

which holds for all $\nu=0,1,2, \ldots$, and $\varphi \in C_{0}^{\infty}\left(B_{r}\right)$ (see [11, Proposition $4.2($ ii)] $)$.

Therefore, for all $\phi=\left(\phi_{1}, \phi_{2}, \phi_{3}\right) \in C_{0}^{\infty}\left(B_{r}\right)$, we find

$$
\begin{aligned}
\left|\left\langle G_{i j}^{N}-G_{i j}^{M}, \phi\right\rangle\right| & =\left|\left\langle\left(\Psi_{N}-\Psi_{M}\right) U_{i} U_{j},-\operatorname{div} \mathbf{I}_{2}(\phi)\right\rangle\right| \\
& \leq c r^{\frac{3-\gamma}{2}}\|\phi\|_{L^{2}\left(B_{r}\right)} \sum_{\nu=N_{0}}^{\infty} 2^{-\frac{\nu \gamma}{2}} .
\end{aligned}
$$

That is, by duality

$$
\left\|G_{i j}^{N}-G_{i j}^{M}\right\|_{\mathcal{M}^{2, \gamma}\left(\mathbb{R}^{3}\right)} \leq c \sum_{\nu=N_{0}}^{\infty} 2^{-\frac{\nu \gamma}{2}} \rightarrow 0 \text { as } M, N \rightarrow+\infty .
$$

A similar (and simpler) argument also shows that $G_{i, j}^{N} \in \mathcal{M}^{2, \gamma}\left(\mathbb{R}^{3}\right)$ for all $N \geq 1$. Thus $\left\{G_{i j}^{N}\right\}$ is a Cauchy sequence in $\mathcal{M}^{2, \gamma}\left(\mathbb{R}^{3}\right)$ which converges to a limit $G_{i, j}$ as claimed in (3.5).

We are now ready to construct the desired pressure $P$.

Lemma 3.2. Suppose that $U$ is a weak solution of (1.3) such that (1.6) holds for some $0<\gamma \leq 3$. Let $R_{j}=\partial_{j}(-\Delta)^{\frac{1}{2}}$ be the $j$-th Riesz transform for $j=1,2,3$. Define a distribution $P$ by letting

$$
\langle P, \varphi\rangle=\left\langle R_{i} R_{j}\left(G_{i j}\right), \nabla \varphi\right\rangle, \quad \varphi \in C_{0}^{\infty}\left(\mathbb{R}^{3}\right),
$$

where $G_{i j}=\left(G_{i j}^{1}, G_{i j}^{2}, G_{i j}^{3}\right) \in \mathcal{M}^{2, \gamma}\left(\mathbb{R}^{3}\right)$ is given by Lemma 3.1, and

$$
R_{i} R_{j}\left(G_{i j}\right)=\left(R_{i} R_{j}\left(G_{i j}^{1}\right), R_{i} R_{j}\left(G_{i j}^{2}\right), R_{i} R_{j}\left(G_{i j}^{3}\right)\right)
$$


Then $P$ satisfies the following growth estimate

$$
\|P\|_{L^{-1,2}\left(B_{r}(x)\right)} \leq C r^{\frac{3-\gamma}{2}}, \quad \forall x \in \mathbb{R}^{3}, r>0 .
$$

Moreover, $P$ satisfies (3.1) and $(U, P)$ smoothly solves

$$
-\nu \Delta U+a U+a(y \cdot \nabla) U+(U \cdot \nabla) U+\nabla P=0 \quad \text { in } \mathbb{R}^{3} .
$$

Proof. By Lemma 3.1, the vector fields $G_{i j} \in \mathcal{M}^{2, \gamma}\left(\mathbb{R}^{3}\right)$. Since $R_{i} R_{j}$ is bounded on $\mathcal{M}^{2, \gamma}\left(\mathbb{R}^{3}\right.$ ) (see, e.g., 13) this implies that $P$ is well-defined and (3.8) holds. Indeed, for any $\varphi \in C_{0}^{\infty}\left(B_{r}(x)\right)$ we have

$$
\begin{aligned}
|\langle P, \varphi\rangle| & =\left|\int_{B_{r}(x)}\left(R_{i} R_{j}\left(G_{i j}\right)\right) \cdot \nabla \varphi d y\right| \\
& \leq\left\|R_{i} R_{j}\left(G_{i j}\right)\right\|_{L^{2}\left(B_{r}(x)\right)}\|\nabla \varphi\|_{L^{2}\left(B_{r}(x)\right)} \\
& \leq C\left\|G_{i j}\right\|_{\mathcal{M}^{2, \gamma}\left(\mathbb{R}^{3}\right)} r^{\frac{3-\gamma}{2}}\|\nabla \varphi\|_{L^{2}\left(B_{r}(x)\right)},
\end{aligned}
$$

which obviously yields (3.8).

Using the facts that $-\operatorname{div} G_{i j}=U_{i} U_{j}$ and $R_{i} R_{j} \Delta \varphi=-\partial_{i} \partial_{j} \varphi$ for any $\varphi \in C_{0}^{\infty}\left(\mathbb{R}^{3}\right)$ (see [15, p. 59]) we can now calculate

$$
\begin{aligned}
\langle\Delta P, \varphi\rangle & =\int_{\mathbb{R}^{3}}\left(R_{i} R_{j}\left(G_{i j}\right)\right) \cdot \nabla \Delta \varphi d x \\
& =\int_{\mathbb{R}^{3}} G_{i j} \cdot \nabla R_{i} R_{j}(\Delta \varphi) d x \\
& =\int_{\mathbb{R}^{3}} U_{i} U_{j} R_{i} R_{j}(\Delta \varphi) d x \\
& =-\int_{\mathbb{R}^{3}} U_{i} U_{j} \partial_{i} \partial_{j} \varphi d x .
\end{aligned}
$$

That is, $P$ is a distributional solution of (3.1) and thus by Weyl's lemma it is smooth (since $U_{i} U_{j}$ is smooth). Note that the second equality in (3.10) requires an explanation as in general $\left|G_{i j}\right| \notin L^{2}\left(\mathbb{R}^{3}\right)$ unless $\gamma=3$. But since $\left|G_{i j}\right| \in \mathcal{M}^{2, \gamma}\left(\mathbb{R}^{3}\right)$ we have $\left|G_{i j}\right| \in L_{\text {loc }}^{2}\left(\mathbb{R}^{3}\right)$ and moreover

$$
\int_{\mathbb{R}^{3}}\left|G_{i j}\right|^{2}\left(1+|x|^{2}\right)^{-\frac{\mu}{2}} d x \leq c\left\|G_{i j}\right\|_{\mathcal{M}^{2, \gamma}}^{2}<+\infty .
$$

for any $\mu>3-\gamma$. Inequality (3.11) can be found in [8], page 132 .

Now let $\chi_{B_{R}(0)}$ be the characteristic function of $B_{R}(0)$. Using Hölder's inequality we have

$$
\begin{aligned}
& \left|\int_{\mathbb{R}^{3}}\left(R_{i} R_{j}\left(G_{i j}\right)-R_{i} R_{j}\left(\chi_{B_{R}(0)} G_{i j}\right)\right) \cdot \nabla \Delta \varphi d x\right| \\
& \quad \leq\left(\int_{\mathbb{R}^{3}}\left|R_{i} R_{j}\left(G_{i j}-\chi_{B_{R}(0)} G_{i j}\right)\right|^{2} w(x) d x\right)^{\frac{1}{2}}\left(\int_{\mathbb{R}^{3}}|\nabla \Delta \varphi|^{2} w(x)^{-1} d x\right)^{\frac{1}{2}},
\end{aligned}
$$

where we choose the weight $w(x)=\left(1+|x|^{2}\right)^{-\frac{\mu}{2}}$. Note that $w$ belongs to the Muckenhoupt $A_{2}$ class provided we choose $\mu \in(3-\gamma, 3)$ (see, e.g., [16, 
Chap. V]). Since $R_{i} R_{j}$ is bounded on the weighted space $L_{w}^{2}\left(\mathbb{R}^{3}\right)$ (see [16, p. 205]) we then have

$$
\begin{aligned}
& \left|\int_{\mathbb{R}^{3}}\left(R_{i} R_{j}\left(G_{i j}\right)-R_{i} R_{j}\left(\chi_{B_{R}(0)} G_{i j}\right)\right) \cdot \nabla \Delta \varphi d x\right| \\
& \quad \leq C\left(\int_{\mathbb{R}^{3}}\left|G_{i j}-\chi_{B_{R}(0)} G_{i j}\right|^{2} w(x) d x\right)^{\frac{1}{2}} .
\end{aligned}
$$

Thus using (3.11) and the Lebesgue's dominated convergence theorem, we eventually find

$$
\begin{aligned}
\int_{\mathbb{R}^{3}}\left(R_{i} R_{j}\left(G_{i j}\right)\right) \cdot \nabla \Delta \varphi d x & =\lim _{R \rightarrow+\infty} \int_{\mathbb{R}^{3}}\left(R_{i} R_{j}\left(\chi_{B_{R}(0)} G_{i j}\right)\right) \cdot \nabla \Delta \varphi d x \\
& =\lim _{R \rightarrow+\infty} \int_{\mathbb{R}^{3}}\left(\chi_{B_{R}(0)} G_{i j}\right) \cdot R_{i} R_{j}(\nabla \Delta \varphi) d x \\
& =\int_{\mathbb{R}^{3}} G_{i j} \cdot \nabla R_{i} R_{j}(\Delta \varphi) d x,
\end{aligned}
$$

as desired. Here the second equality follows since $\chi_{B_{R}(0)} G_{i j} \in L^{2}\left(\mathbb{R}^{3}\right)$, and the last equality follows since $R_{i} R_{j}(\nabla \Delta \varphi)=\nabla R_{i} R_{j}(\Delta \varphi)$ has compact support.

Finally, to prove (3.9), we let

$$
F:=-\nu \Delta U+a U+a(y \cdot \nabla) U+(U \cdot \nabla) U+\nabla P
$$

and show that $F \equiv 0$.

Using (2.3) with appropriate test functions $\phi$ we find $\operatorname{curl} F=0$. Also, by (3.1) and the fact that $\operatorname{div} U=0$ we have $\operatorname{div} F=0$. These imply that $\Delta F=0$. Thus by the mean-value property of harmonic functions we find

$$
F(0)=\epsilon^{3} \int_{\mathbb{R}^{3}} F(y) \varphi(\epsilon y) d y
$$

for any $\epsilon>0$ and every radial function $\varphi \in C_{0}^{\infty}\left(B_{1}(0)\right)$ such that $\int_{\mathbb{R}^{3}} \varphi d y=$ 1 (see [15, p. 275]).

Following [12, by analyticity, to show that $F \equiv 0$ it is enough to verify that $D^{\alpha} F(0)=0$ for each for each multi-index $\alpha=\left(\alpha_{1}, \alpha_{2}, \alpha_{3}\right)$ with $|\alpha| \geq 0$. To this end, we first apply (3.12) to the harmonic function $D^{\alpha} F$ and then integrate by parts to obtain

$$
D^{\alpha} F(0)=\epsilon^{3} \int_{\mathbb{R}^{3}} D^{\alpha} F(y) \varphi(\epsilon y) d y=(-1)^{|\alpha|} \epsilon^{3} \int_{\mathbb{R}^{3}} F(y) \epsilon^{|\alpha|}\left(D^{\alpha} \varphi\right)(\epsilon y) d y,
$$

where $\epsilon$ and $\varphi$ are as above. Thus to show that $D^{\alpha} F(0)=0$, it is enough to verify that

$$
\lim _{\epsilon \rightarrow 0^{+}} \epsilon^{3} \int_{\mathbb{R}^{3}} F(y) \theta(\epsilon y) d y=0
$$


for any function $\theta \in C_{0}^{\infty}\left(B_{1}(0)\right)$. That is, we need to show that

$$
\lim _{\epsilon \rightarrow 0^{+}} \epsilon^{3} \int_{\mathbb{R}^{3}}[-\nu \Delta U+a U+a(y \cdot \nabla) U+(U \cdot \nabla) U+\nabla P] \theta(\epsilon y) d y=0 .
$$

The first four terms in the above expression can be treated similarly. For example, for the term involving $a(y \cdot \nabla) U$ by integrating by parts we have

$$
\begin{aligned}
\epsilon^{3} & \int(y \cdot \nabla) U(y) \theta(\epsilon y) d y \\
& =-\epsilon^{3} \int 3 U(y) \theta(\epsilon y) d y-\epsilon^{3} \int U(y)\left(\epsilon y_{i}\right)\left(\partial_{i} \theta\right)(\epsilon y) d y \\
& =-3 \epsilon^{3} \int U(y) \theta(\epsilon y) d y-\epsilon^{3} \int U(y) \widetilde{\theta}(\epsilon y) d y,
\end{aligned}
$$

where $\widetilde{\theta}(y)=\left(\epsilon y_{i}\right)\left(\partial_{i} \varphi\right)(y)$.

On the other hand, by Hölder's inequality and a simple change of variables we find

$$
\begin{aligned}
\left|\int_{\mathbb{R}^{3}} U(y) \theta(\epsilon y) d y\right| & \leq\left(\int_{B_{1 / \epsilon}}|U|^{2}(y)|\theta(\epsilon y)| d y\right)^{\frac{1}{2}}\left(\int_{B_{1 / \epsilon}}|\theta(\epsilon y)| d y\right)^{\frac{1}{2}} \\
& \leq \epsilon^{-\frac{3}{2}}\left(\int_{B_{1 / \epsilon}}|U|^{2}(y)|\theta(\epsilon y)| d y\right)^{\frac{1}{2}}\left(\int_{B_{1}}|\theta(z)| d z\right)^{\frac{1}{2}} \\
& \leq c \epsilon^{-\frac{3}{2}}\left(\int_{B_{1 / \epsilon}}|U|^{2}(y)|\theta(\epsilon y)| d y\right)^{\frac{1}{2}} .
\end{aligned}
$$

Thus by (2.2) it holds that

$$
\begin{aligned}
& \left|\int_{\mathbb{R}^{3}} U(y) \theta(\epsilon y) d y\right| \\
& \leq c \epsilon^{-\frac{3}{2}}\left(\left\||U|^{2}\right\|_{L^{-1,2}\left(B_{1 / \epsilon}\right)}\right)^{\frac{1}{2}}\left(\int_{B_{1 / \epsilon}}|\epsilon(\nabla|\theta|)(\epsilon y)|^{2} d y\right)^{\frac{1}{4}} \\
& \quad \leq c \epsilon^{-\frac{3}{2}+\frac{1}{2}}\left(\int_{B_{1 / \epsilon}}\left|\mathbf{I}_{1}\left(|U|^{2}\right)\right|^{2} d y\right)^{\frac{1}{4}}\left(\int_{B_{1 / \epsilon}}|(\nabla \theta)(\epsilon y)|^{2} d y\right)^{\frac{1}{4}} \\
& \quad \leq c \epsilon^{-1-\frac{3}{4}}\left(\int_{B_{1 / \epsilon}}\left|\mathbf{I}_{1}\left(|U|^{2}\right)\right|^{2} d y\right)^{\frac{1}{4}}\left(\int_{B_{1}}|\nabla \theta(z)|^{2} d z\right)^{\frac{1}{4}},
\end{aligned}
$$

which by our assumption on $U$ yields

$$
\left|\int_{\mathbb{R}^{3}} U(y) \theta(\epsilon y) d y\right| \leq c \epsilon^{-\frac{7}{4}}(1 / \epsilon)^{\frac{3-\gamma}{4}}=c \epsilon^{-\frac{5}{2}+\frac{\gamma}{4}} .
$$


Of course, the same inequality also holds with $\tilde{\theta}$ in place of $\theta$. Hence, using these in the expression for $\epsilon^{3} \int(y \cdot \nabla) U(y) \theta(\epsilon y) d y$ above we obtain

$$
\left|\epsilon^{3} \int(y \cdot \nabla) U(y) \theta(\epsilon y) d y\right| \leq c \epsilon^{\frac{1}{2}+\frac{\gamma}{4}} \rightarrow 0 \text { as } \epsilon \rightarrow 0 .
$$

For what concerns the term involving $\nabla P$ in (3.13), using integration by parts and the bound (3.8) we have

$$
\begin{aligned}
\left|\epsilon^{3} \int \nabla P \theta(\epsilon y) d y\right| & =\left|-\epsilon^{4} \int P(y)(\nabla \theta)(\epsilon y) d y\right| \\
& \leq c \epsilon^{4}\|P\|_{L^{-1,2}\left(B_{1 / \epsilon}\right)}\left(\int_{B_{1 / \epsilon}}\left|\epsilon\left(\nabla^{2} \theta\right)(\epsilon y)\right|^{2} d y\right)^{\frac{1}{2}} \\
& \leq c \epsilon^{5}(1 / \epsilon)^{\frac{3-\gamma}{2}}\left(\int_{B_{1 / \epsilon}}\left|\left(\nabla^{2} \theta\right)(\epsilon y)\right|^{2} d y\right)^{\frac{1}{2}} \\
& \leq c \epsilon^{5-\frac{3}{2}-\frac{3}{2}+\frac{\gamma}{2}}\left(\int_{B_{1}}\left|\nabla^{2} \theta(z)\right|^{2} d z\right)^{\frac{1}{2}} .
\end{aligned}
$$

Thus we also have

$$
\left|\epsilon^{3} \int \nabla P \theta(\epsilon y) d y\right| \rightarrow 0 \text { as } \epsilon \rightarrow 0 .
$$

In conclusion, we obtain (3.13) and that completes the proof of the lemma.

\section{Proof of Theorem 1.4}

We are now ready to prove Theorem 1.4 .

Proof of Theorem 1.4. Obviously, it is enough to prove the first statement of the theorem. Henceforth, suppose that $U$ is a weak solution of (1.3) such that condition (1.6) holds for some $\gamma \in(0,3]$. Let the function $P$ be defined as in Lemma 3.2. Then $(U, P)$ smoothly solves (1.3), and thus the functions

$$
u(x, t)=\lambda(t) U(\lambda(t) x), \quad \text { and } \quad p(x, t)=\lambda^{2}(t) P(\lambda(t) x),
$$

with $\lambda(t)=[2 a(T-t)]^{-1 / 2}, T>0$, solves the Navier-Stokes equations (1.1) in the classical sense in $\mathbb{R}^{3} \times(-\infty, T)$. By Theorem 1.1, it is enough to check that

$$
\underset{T-1<t<T}{\operatorname{ess} \sup _{B_{1}(0)}} \frac{1}{2}|u(x, t)|^{2} d x+\int_{T-1}^{T} \int_{B_{1}(0)} \nu|\nabla u(x, t)|^{2} d x d t<+\infty .
$$

To this end, we first observe that for any ball $B_{r}\left(x_{0}\right) \subset \mathbb{R}^{3}$ and $t<T$, it holds that

$$
\left\||u(\cdot, t)|^{2}\right\|_{L^{-1,2}\left(B_{r}\left(x_{0}\right)\right)}+\|p(\cdot, t)\|_{L^{-1,2}\left(B_{r}\left(x_{0}\right)\right)} \leq c \lambda(t)^{1-\frac{\gamma}{2}} r^{\frac{3-\gamma}{2}} .
$$


Indeed for any $\varphi \in C_{0}^{\infty}\left(B_{r}\left(x_{0}\right)\right)$ and with $\lambda=\lambda(t)$ we have

$$
\begin{aligned}
\left.\left|\int_{B_{r}\left(x_{0}\right)}\right| u(x, t)\right|^{2} \varphi(x) d x \mid & =\left.\lambda^{2}\left|\int_{B_{r}\left(x_{0}\right)}\right| U(\lambda x)\right|^{2} \varphi(x) d x \mid \\
& =\left.\lambda^{-1}\left|\int_{B_{r \lambda}\left(\lambda x_{0}\right)}\right| U(z)\right|^{2} \varphi(z / \lambda) d z \mid .
\end{aligned}
$$

From this using inequality (2.2) we obtain

$$
\begin{aligned}
& \left.\left|\int_{B_{r}\left(x_{0}\right)}\right| u(x, t)\right|^{2} \varphi(x) d x \mid \\
& \quad \leq c \lambda^{-1}|||U|^{2} \|_{L^{-1,2}\left(B_{r \lambda}\left(\lambda x_{0}\right)\right)}\left(\int_{B_{r \lambda}\left(\lambda x_{0}\right)}\left|\frac{1}{\lambda} \nabla \varphi\left(\frac{z}{\lambda}\right)\right|^{2} d z\right)^{\frac{1}{2}} \\
& \quad \leq c \lambda^{-2}(r \lambda)^{\frac{3-\gamma}{2}}\left(\int_{B_{r}\left(x_{0}\right)}|\nabla \varphi(x)|^{2} \lambda^{3} d x\right)^{\frac{1}{2}} \\
& \quad \leq \lambda^{1-\frac{\gamma}{2}} r^{\frac{3-\gamma}{2}}\left(\int_{B_{r}\left(x_{0}\right)}|\nabla \varphi(x)|^{2} d x\right)^{\frac{1}{2}} .
\end{aligned}
$$

This gives

$$
\left\||u(\cdot, t)|^{2}\right\|_{L^{-1,2\left(B_{r}\left(x_{0}\right)\right)}} \leq c \lambda(t)^{1-\frac{\gamma}{2}} r^{\frac{3-\gamma}{2}} .
$$

Likewise, using the bound (3.8) and an analogous argument we obtain a similar bound for $p$. Thus (4.2) is proved.

Next we shall make use of the well-known energy equality:

$$
\begin{aligned}
& \int_{B_{R}}|u(x, t)|^{2} \phi(x, t) d x+2 \nu \int_{T_{1}}^{t} \int_{B_{R}}|\nabla u|^{2} \phi(x, s) d x d s \\
& =\int_{T_{1}}^{t} \int_{B_{R}}|u|^{2}\left(\phi_{t}+\nu \Delta \phi\right) d x d s+\int_{T_{1}}^{t} \int_{B_{R}}\left(|u|^{2}+2 p\right) u \cdot \nabla \phi d x d s,
\end{aligned}
$$

which holds for every ball $B_{R}=B_{R}(0), t \in\left(T_{1}, T\right)$, and any nonnegative function $\phi \in C_{0}^{\infty}\left(\mathbb{R}^{3} \times \mathbb{R}\right)$ vanishing in a neighborhood of the parabolic boundary $B_{R} \times\left\{t=T_{1}\right\} \cup \partial B_{R} \times\left[T_{1}, T\right]$ of the cylinder $B_{R} \times\left(T_{1}, T\right)$.

Let $T_{\epsilon}=T-\epsilon$ for sufficiently small $\epsilon>0$, say, $\epsilon \in(1 / 2,0)$. For any balls

$$
B_{s}=B_{s}(0), \quad B_{\rho}=B_{\rho}(0),
$$

with $1 \leq s<\rho \leq 2$, we consider a test function $\phi(x, t)=\eta_{1}(x) \eta_{2}(t)$ where $\eta_{1} \in C_{0}^{\infty}\left(B_{\rho}\right), 0 \leq \eta_{1} \leq 1$ in $\mathbb{R}^{n}, \eta_{1} \equiv 1$ on $B_{s}$, and

$$
\left|\nabla^{\alpha} \eta_{1}\right| \leq \frac{c}{(\rho-s)^{|\alpha|}}
$$


for all multi-indices $\alpha$ with $|\alpha| \leq 3$. The function $\eta_{2}(t)$ is chosen so that $\eta_{2} \in C_{0}^{\infty}\left(T_{\epsilon}-\rho^{2}, T_{\epsilon}+\rho^{2}\right), 0 \leq \eta_{2} \leq 1$ in $\mathbb{R}, \eta_{2}(t) \equiv 1$ for $t \in\left[T_{\epsilon}-s^{2}, T_{\epsilon}+s^{2}\right]$, and

$$
\left|\eta_{2}^{\prime}(t)\right| \leq \frac{c}{\rho^{2}-s^{2}} \leq \frac{c}{\rho-s}
$$

Thus

$$
\left|\nabla \phi_{t}\right|+|\nabla \Delta \phi| \leq \frac{c}{(\rho-s)^{3}}, \quad\left|\nabla^{2} \phi\right| \leq \frac{c}{(\rho-s)^{2}}, \quad|\nabla \phi| \leq \frac{c}{\rho-s} .
$$

Let

$$
I(s, \epsilon)=\sup _{T_{\epsilon}-s^{2} \leq t \leq T_{\epsilon}} \int_{B_{s}}|u(x, t)|^{2} d x+\int_{T_{\epsilon}-s^{2}}^{T_{\epsilon}} \int_{B_{s}}|\nabla u(x, t)|^{2} d x d t,
$$

which is a finite quantity provided $\epsilon>0$.

Using $\phi$ as a test function in the energy equality above we have

$$
\begin{aligned}
I(s, \epsilon) \leq & c \int_{T_{\epsilon}-\rho^{2}}^{T_{\epsilon}}\left\|\left.u\right|^{2}\right\|_{L^{-1,2}\left(B_{\rho}\right)}\left\|\nabla \phi_{t}+\nabla \Delta \phi\right\|_{L^{2}\left(B_{\rho}\right)} d t+ \\
& +c \int_{T_{\epsilon}-\rho^{2}}^{T_{\epsilon}}\left\|\left.u\right|^{2}+2 p\right\|_{L^{-1,2}\left(B_{\rho}\right)}\left\|\nabla u \cdot \nabla \phi+u \cdot \nabla^{2} \phi\right\|_{L^{2}\left(B_{\rho}\right)} d t \\
\leq & c \int_{T_{\epsilon}-\rho^{2}}^{T_{\epsilon}} \lambda(t)^{1-\frac{\gamma}{2}}\left\|\nabla \phi_{t}+\nabla \Delta \phi\right\|_{L^{2}\left(B_{\rho}\right)} d t+ \\
& +c \int_{T_{\epsilon}-\rho^{2}}^{T_{\epsilon}} \lambda(t)^{1-\frac{\gamma}{2}}\left\|\nabla u \cdot \nabla \phi+u \cdot \nabla^{2} \phi\right\|_{L^{2}\left(B_{\rho}\right)} d t,
\end{aligned}
$$

where we used (4.2) and the fact that $1 \leq \rho \leq 2$. From the choice of $\phi$, this gives

$$
\begin{aligned}
I(s, \epsilon) \leq & \frac{c}{(\rho-s)^{3}} \int_{T_{\epsilon}-\rho^{2}}^{T_{\epsilon}} \lambda(t)^{1-\frac{\gamma}{2}} d t+ \\
& +c \int_{T_{\epsilon}-\rho^{2}}^{T_{\epsilon}} \lambda(t)^{1-\frac{\gamma}{2}}\left(\frac{\|\nabla u\|_{L^{2}\left(B_{\rho}\right)}}{\rho-s}+\frac{\|u\|_{L^{2}\left(B_{\rho}\right)}}{(\rho-s)^{2}}\right) d t .
\end{aligned}
$$

Thus by Hölder's inequality we find

$$
\begin{aligned}
& I(s, \epsilon) \leq \frac{c}{(\rho-s)^{3}} \int_{T_{\epsilon}-\rho^{2}}^{T_{\epsilon}} \lambda(t)^{1-\frac{\gamma}{2}} d t+ \\
& +\frac{c}{\rho-s}\left(\int_{T_{\epsilon}-\rho^{2}}^{T_{\epsilon}} \lambda(t)^{2-\gamma} d t\right)^{\frac{1}{2}}\left(\int_{T_{\epsilon}-\rho^{2}}^{T_{\epsilon}}\|\nabla u\|_{L^{2}\left(B_{\rho}\right)}^{2} d t\right)^{\frac{1}{2}}+ \\
& +\frac{c}{(\rho-s)^{2}}\left(\int_{T_{\epsilon}-\rho^{2}}^{T_{\epsilon}} \lambda(t)^{2-\gamma} d t\right)^{\frac{1}{2}}\left(\sup _{T_{\epsilon}-\rho^{2} \leq t \leq T_{\epsilon}}\|u(\cdot, t)\|_{L^{2}\left(B_{\rho}\right)}^{2}\right)^{\frac{1}{2}} .
\end{aligned}
$$


Now using Young's inequality we arrive at

$$
\begin{aligned}
I(s, \epsilon) \leq & \frac{c}{(\rho-s)^{3}} \int_{T_{\epsilon}-\rho^{2}}^{T_{\epsilon}} \lambda(t)^{1-\frac{\gamma}{2}} d t+ \\
& +\left(\frac{c}{(\rho-s)^{2}}+\frac{c}{(\rho-s)^{4}}\right) \int_{T_{\epsilon}-\rho^{2}}^{T_{\epsilon}} \lambda(t)^{2-\gamma} d t+\frac{1}{2} I(\rho, \epsilon)
\end{aligned}
$$

As this holds for all $1 \leq s<\rho \leq 2$ by Lemma 2.1 we find

$$
\begin{aligned}
I(1, \epsilon) & \leq C \int_{T_{\epsilon}-4}^{T_{\epsilon}} \lambda(t)^{1-\frac{\gamma}{2}} d t+C \int_{T_{\epsilon}-4}^{T_{\epsilon}} \lambda(t)^{2-\gamma} d t \\
& \leq C \int_{T-5}^{T}\left[\lambda(t)^{1-\frac{\gamma}{2}}+\lambda(t)^{2-\gamma}\right] d t \\
& \leq C(a, \gamma)<+\infty .
\end{aligned}
$$

Since this holds for every $\epsilon \in(1 / 2,0)$ we deduce that (4.1) holds and thus the proof is complete.

\section{REFERENCES}

[1] D. R. Adams. A note on Riesz potentials. Duke Math. J., 42(4):765-778, 1975.

[2] L. Caffarelli, R. Kohn, and L. Nirenberg. Partial regularity of suitable weak solutions of the Navier-Stokes equations. Comm. Pure Appl. Math., 35(6):771-831, 1982.

[3] J. Frehse and M. Růžička. On the regularity of the stationary Navier-Stokes equations. Ann. Sc. Norm. Pisa, 21:63-95, 1994.

[4] J. Frehse and M. Růžička. Regularity of the stationary Navier-Stokes equations in bounded domains. Arch. Rat. Mech. Anal. 128:361-381, 1994.

[5] G. Galdi. An introduction to the mathematical theory of Navier-Stokes equations, I, II. Springer, 1994.

[6] M. Giaquinta and G. Modica. Nonlinear systems of the type of the stationary NavierStokes system. J. Reine Angew. Math., 330:173-214, 1982.

[7] E. Giusti. Direct methods in the calculus of variations. World Scientific Publishing Co., Inc., River Edge, NJ, 2003.

[8] T. Kato. Strong solutions of the Navier-Stokes equation in Morrey spaces. Bol. Soc. Brasil. Mat. (N.S.), 22(2):127-155, 1992.

[9] O. A. Ladyzhenskaya. The mathematical theory of viscous incompressible flow, 2nd edition. Gordon and Breach, New York, 1969.

[10] J. Leray. Sur le mouvement d'un liquide visqueux emplissant l'espace. Acta Math., 63(1):193-248, 1934.

[11] V. G. Maz'ya and I. E. Verbitsky. Form boundedness of the general second-order differential operator. Comm. Pure Appl. Math., 59(9):1286-1329, 2006.

[12] J. Nečas, M. Růžička, and V. Šverák. On Leray's self-similar solutions of the NavierStokes equations. Acta Math., 176(2):283-294, 1996.

[13] J. Peetre. On convolution operators leaving $L^{p, \lambda}$ spaces invariant. Ann. Mat. Pura Appl., 72(4):295-304, 1966.

[14] N. C. Phuc. The Navier-Stokes equations in nonendpoint borderline Lorentz spaces. J. Math. Fluid Mech., 17: 741-760, 2015.

[15] E. M. Stein. Singular integrals and differentiability properties of functions. Princeton Math. Ser., 30. Princeton Univ. Press, Princeton, N J, 1970.

[16] E. M. Stein. Harmonic analysis: real-variable methods, orthogonality, and oscillatory integrals. Princeton University Press, 1993. 
[17] R. Temam. Navier-Stokes equations: theory and numerical analysis. North-Holland, Amsterdam and New York, 1977.

[18] T.-P. Tsai. On Leray's self-similar solutions of the Navier-Stokes equations satisfying local energy estimates. Arch. Rat. Mech. Anal., 143(1):29-51, 1998.

[19] M. Struwe. Regular solutions of the stationary Navier-Stokes equations on $\mathbb{R}^{5}$. Math. Ann., 302:719-741, 1995.

Department of Mathematics, Louisiana State University, 303 Lockett Hall, Baton Rouge, LA 70803, USA.

E-mail address: cguevara@lsu.edu

Department of Mathematics, Louisiana State University, 303 Lockett Hall, Baton Rouge, LA 70803, USA.

E-mail address: pcnguyen@math.1su.edu 